\title{
German Literature as World Literature, hrsg. von Thomas $O$. Beebee
}

http://dx.doi.org/10.1590/1982-88371841875

Heike Muranyi ${ }^{1}$

BEEBEE, Thomas O. (Hrsg.). German Literature as World Literature. New York u. London: Bloomsbury, 2014. ISBN 978-1-62356-391-2.

Mit German Literature as World Literature legt Thomas O. Beebee eine Aufsatzsammlung vor, die einen so sinn- wie überfälligen Blickwinkel auf die deutsche Literatur - d. h. der literarischen Produktion in deutscher Sprache - einnimmt. Mit David DAMrosch (2003) davon ausgehend, dass es sich bei Weltliteratur um eine „elliptical refraction of national literature“ handelt, untersucht der Band deutsche Literatur an ihren Schnittstellen $\mathrm{zu}$ anderen Literaturen und Rezeptionskulturen. In Anbetracht der hohen Spezifik der Einzelbeiträge richtet sich dieses Buch vornehmlich an den germanistisch interessierten und versierten Leser - fortgeschrittene Studenten und Wissenschaftler -, der mit der Problematik und Komplexität des Weltliteraturbegriffs bereits vertraut ist. Die Form des Sammelbands kommt dabei der begrifflichen Komplexität entgegen, wie Beebee in seiner ausführlichen und äußerst lesenswerten Einführung erklärt: „The capaciousness, as well as the polylingual and multicultural features of WL present formidable obstacles to its study, and call for collaborative approaches that conjoin a range of expertise.“(11).

War die Verwendung des deutschen Begriffs der Weltliteratur im internationalen literaturwissenschaftlichen Diskurs längst etabliert und die Wichtigkeit deutscher Beiträge $\mathrm{zu}$ seiner - traditionell in der Komparatistik verorteten - theoretischen Diskussion unangefochten, so ließ eine akademische Erörterung und Exemplifizierung

\footnotetext{
${ }^{1}$ Universidade Federal do Paraná (UFPR), Setor de Ciências Humanas, DEPAC, Archiv.br, Rua General Carneiro, 460, Edifício D. Pedro I - $10^{\circ}$ andar, 80.060-150, Curitiba, PR, Brasil. Email: heike.muranyi@archivbr.de
} 
Muranyi, H. - German Literature as World Literature

der Anwendbarkeit des Begriffs auf die deutsche Literatur in größerem Umfang bislang auf sich warten.

In drei Kategorien ("Goethe's Weltliteratur/World Literature”, "Ausstrahlungen/Emanations" und "Schnittmengen/Intersections") belegen die insgesamt neun Beiträge auf beeindruckende Weise, wie vielfältig sich eine germanistische, literaturwissenschaftliche Forschung unter dem Vorzeichen des Weltliteraturbegriffs gestalten lässt. Damit leistet German Literature as World Literature nicht nur einen Beitrag zur germanistischen Literaturforschung als solche, sondern steuert auch zu einer Begriffsdebatte bei, welche in Zeiten von Globalisierung und einer damit einhergehenden erhöhten Mobilität sowie zahlreichen neuen Migrationsbewegungen und kulturellen Transferprozessen nicht nur hoch im Kurs stehen, sondern auch und vor allem $\mathrm{zu}$ einer Revision der Inhalte und Deutungsmöglichkeiten der zur Diskussion stehenden Begrifflichkeit aufrufen sollte.

Indem German Literature as World Literature den Schwerpunkt auf die Schnittstellen von nationaler und Weltliteratur legt, sieht sich der Band nicht zuletzt einem komparatistischen Ansatz verpflichtet, der jedoch, wie Beebee in seiner Einführung schreibt, den Fokus stärker auf die den literarischen Traditionen inhärenten Dynamiken richten müsse. Des Weiteren rekapituliert Beebee den deutschen Anteil an der Formung des Konzepts der Weltliteratur, betont die Durchlässigkeit in Beziehung zum kulturellen Anderen als eine Konstante der deutschen Literatur („From the Germania of Tacitus [...] to the emergence of Turkish-German literature and the presence of Russian German writers such as Wladimir Kaminer on the current scene, German literature has been imbricated in other cultural traditions“) und reüssiert schließlich die verschiedenen Deutungen des Weltliteraturbegriffs, wobei er in der Auseinandersetzung mit der Kritik am qualitativen, ,hyperkanonischen' Weltliteraturbegriff zwar sehr stimmig mit Durs Grünbein argumentiert, auf einen für die (komparatistische) Diskussion grundlegenden und sicherlich auch noch heute lesenswerten theoretischen Beitrag von René ÉTIEMBLE („Faut-il réviser la notion de Weltliteratur?") jedoch nicht verweist.

Geradezu als Reverenz an den unbestrittenen Gründervater der Diskussion widmen sich die ersten beiden Beiträge des Bandes der Goethischen Lesart des Weltliteraturbegriffs. Chunjie ZHANG beleuchtet in ihrem Aufsatz Goethes Rezeption 
Muranyi, H. - German Literature as World Literature

chinesischer Literatur und kommt bei ihrem Vergleich der weiblichen Hauptfiguren der Wahlverwandtschaften (1809) und dem erst 1832 ins Englische übersetzten Roman von Cao Xuequin, The Story of the Stone (1791) - d.h. zwischen zwei intertextuell nicht direkt miteinander in Beziehung stehenden Texten - zu erstaunlichen Ergebnissen. Auch Daniel PuRDY setzt sich mit Goethes Beziehungen zur chinesischen Literatur auseinander, wobei hier die Rezeption des von Abel Rémusat aus dem Chinesischen übertragenen Romans Les Deux Cousines im Vordergrund der Betrachtungen steht.

Die Beiträge des zweiten Teils beschäftigen sich mit deutschsprachigen Autoren unterschiedlichster Couleur und Provenienz und der weltliterarischen Verfasstheit ihres Schaffens. Ihnen gemeinsam ist, dass sie sich entweder im Spannungsfeld multiethnischer, mehrsprachiger Gemeinschaften $\mathrm{zu}$ Autoren entwickelten (Hofmannsthal, Rilke) oder die Erfahrung des Exils für die Genese und internationalen Rezeption ihrer Werke von Bedeutung war (Brecht, Sebald). Hugo von Hofmannsthal und die ethnischen und kulturellen Konflikte in der Donaumonarchie sind Gegenstand der Untersuchungen von Simona MOTI; Kathleen KOMAR beschäftigt Rainer Maria Rilkes Position im Hyperkanon und verweist dabei auf die paradoxe Verknüpfung des (sprachlich) Verorteten und der universalen Bedeutsamkeit: Kaum ein Lyriker deutscher Sprache ist den Feinheiten und Komplexitäten seiner Muttersprache so sehr verpflichtet und verbunden, kaum ein anderer wurde zugleich so weitreichend übersetzt und rezipiert wie Rilke. Martina KOLB stellt in ihrem Beitrag Bertolt Brechts Exilbiographie in den Kontext seines Schaffens und dessen weltweiter Rezeption, während David D. KIM sich mit W.G. Sebald einem der wohl außergewöhnlichsten Autoren deutscher Sprache widmet, dessen Exil eine persönliche Reaktion auf die traumatische Erinnerung an 1933-1945 war und seinem Weltbürgertum - und nicht zuletzt seiner Weltliteratur einen überaus melancholischen Anstrich verlieh.

Im letzten Teil des Bandes zeigt Thomas O. BEEBEE in seinem Beitrag „From Nobel to Nothingness“ anhand zweier heute weitestgehend vergessener Autoren deutscher Sprache, Rudolf Eucken und Paul Heyse, dass die Verleihung des Nobelpreises für Literatur nicht unweigerlich Einschreibung des jeweiligen Werkes in den weltliterarischen Kanon führt. Paul NiSSLER wirft einen extensiven Blick auf die Schnittstellen deutscher und lateinamerikanischer Literaturen, wobei er, glücklich gewählt, Alexander von Humboldt an Anfang und Ende seiner mit reichlich bibliographischen Informationen unterfütterten Betrachtungen stellt. 


\section{Muranyi, H. - German Literature as World Literature}

Den Versuch einer Neudefinition des Weltliteraturbegriffs unternimmt schließlich Elke STURM-TRIGONAKI in ihrem den Band schließenden Beitrag; sie geht dabei von der Notwendigkeit aus, sprachlich und kulturell hybriden Texten aus ihrer Kategorisierung als ,Minderheitsliteraturen' innerhalb der Literaturen einer Sprache herauszuhelfen. In Anbetracht der Tatsache, dass sich der von Beebee herausgegebene Band sich zum Vorsatz nimmt, ein in der Literaturwissenschaft ein wenig in den Hintergrund gerücktes Konzept neu zu lesen und anzuwenden, ist es möglicherweise ein wenig bedauernswert, dass sich bis auf den letztgenannten Beitrag keiner mit zeitgenössischer literarischer Produktion in deutscher Sprache unter dem Vorzeichen des Weltliteraturbegriffs befasst.

Eine ausführliche Bibliographie anthologischer und kritischer Werke rundet den Band ab. Dieser hätte hie und da ein wenig sorgfältiger lektoriert werden können, stellt aber insgesamt in Form und Gehalt einen guten Auftakt dessen dar, was er propagiert. Es ist zu hoffen, dass die Initiative Beebees und seiner Kollegen Interesse weckt und in der Folge zu vielen weiteren Studien über das Konzept der Weltliteratur - und dessen Anwendbarkeit in der aktuellen germanistischen Literaturforschung - verhilft.

Recebido em 14/03/2015 Aceito em 21/04/2015 